\title{
Chronic migraine in the first COVID-19 lockdown: the impact of sleep, remote working, and other life/psychological changes
}

\author{
Carmelo Tiberio Currò ${ }^{1}$ (D ) Antonio Ciacciarelli ${ }^{1}$. Chiara Vitale ${ }^{1}$. Enrica Serena Vinci ${ }^{1}$ - Antonio Toscano ${ }^{1}$. \\ Giuseppe Vita ${ }^{1} \cdot$ Giuseppe Trimarchi $^{2} \cdot$ Rosalia Silvestri $^{1} \cdot$ Massimo Autunno $^{1}$
}

Received: 22 May 2021 / Accepted: 26 July 2021 / Published online: 8 August 2021

(c) Fondazione Società Italiana di Neurologia 2021

\begin{abstract}
Aims The objective of this study was to evaluate the impact of the first Italian COVID-19 lockdown on patients with chronic migraine (CM).

Material and methods The study was based on an e-mail survey addressed to CM patients of our headache center. The survey evaluated demographic, life style, sleep, psychological, and migraine features during the COVID-19 lockdown period and the month before. The outcomes were migraine impact on daily life and variation in attack frequency, attack duration, migraine pain intensity, migraine symptomatic drugs use per week, and efficacy.

Results Ninety-two patients completed the survey. During the lockdown period, attack frequency was stable in $40,2 \%$, increased in $33,7 \%$, and reduced in $26,1 \%$ of patients; attack duration was stable in $55,4 \%$, increased in $23,9 \%$, and reduced in $20,7 \%$. Migraine pain was stable or reduced in $65,2 \%$ and increased in $34,8 \%$; number of symptomatic drugs per week was stable in $50 \%$, reduced in $29,3 \%$, and increased in $20,7 \%$; migraine drug efficacy was stable in $73,9 \%$, reduced in $17,4 \%$, and increased in $8,7 \%$. Patients had a HIT-6 score of 64,63 $\pm 8,81$. Significant associations were found with remote working, smoke, education, discontinuation of the therapy performed within headache center, migraine familiarity, sleep, anxiety, perceived stress, concern about future, and COVID-19.

Conclusion During the lockdown, approximately half of the patients had a clinical stability, a quarter an improvement, and another quarter a worsening. We identified different migraine-influencing elements; in particular, the remote working could represent an easy way to ameliorate migraineurs' life.
\end{abstract}

Keywords Chronic migraine $\cdot$ Headache $\cdot$ COVID-19 $\cdot$ Lockdown $\cdot$ Life style $\cdot$ Remote working

\section{Introduction}

Migraine represents a social problem with an enormous disability burden, especially in chronic migraine (CM) [1, 2]. It is influenced by life style and habits such as coffee consumption [3, 4], smoke [5], computer, smartphone, and television use [6]. Sleep quality (SQ) [7, 8], depression, anxiety, and stress [4] have also a significant impact.

Carmelo Tiberio Currò

currocarmelotiberio@gmail.com

1 Department of Clinical and Experimental Medicine, University of Messina, Via Consolare Valeria 1, 98124 Messina, Italy

2 Faculty of Medicine and Surgery, University of Messina, Messina, Italy
The COVID-19 pandemic led the governments to introduce a series of restrictive measures referred as "lockdown." Lockdown represented a revolution for life of many people, it was a stressful condition which forced Italians to stay at home limiting human contact, changing the way to live relations and to work in the context of a pandemic which threatened public health and devastated economy.

The aim of the present study was to evaluate the influence of the first COVID-19 lockdown in Italy on CM patients. We investigated the impact of $\mathrm{CM}$ on daily life during the lockdown and changes in frequency, attack duration, pain intensity, and drugs between this period and the previous month.

Using COVID-19 lockdown as a unique occasion to acquire new insights into this disease, the study evaluated the influence of social habit, family life, work life, mood, $\mathrm{SQ}$, perceived stress, and future concern on CM patients. 


\section{Methods}

The present observational cross-sectional study was based on an e-mail survey addressed to patients suffering from CM followed at our headache center. The survey was an editable file that every patient completed and re-sent to our headache center e-mail. The questionnaire is available on supplementary materials. We also verified and added some migraine information using our headache center archive. The study investigated migraine, sleep, life, and psychological features during the previous month and the Italian COVID19 lockdown period which went from March $9^{\text {th }}, 2020$, to May $3^{\text {rd }}, 2020$. The survey started on April $24^{\text {th }}, 2020$, and closed on May $3^{\text {rd }}, 2020$.

\section{Inclusion criteria}

Patients were selected according to the following criteria:

- CM diagnosis based on International Classification of Headache Disorders, third edition criteria [9]

- Age $\geq 18$ years

- Written informed consent to participate to the study

\section{Survey}

The survey consisted of:

- Demographic and life-style module

- Sleep features module

- Psychological module

- Migraine module

Demographic and life-style module consisted of age, gender, educational qualifications, number of son/daughters, age of sons/daughters, COVID-19 province prevalence, size of the house, rent or mortgage to pay, number of people in house, ratio of house size/number of people, living with parents, quality of home-inhabitant relationship, unemployment, work/study stop, remote working (RW), job loss during COVID-19 pandemic, hours of computer use, variation of computer time use, hours of smartphone use, variation of time smartphone use, hours of Internet use, variation of time internet use, hours of television viewing, variation of time television viewing, number of coffee cups, variation of coffee cups, quality variation of nutrition, variation of meal regularity, smoke, variation of smoking habit, times a day to research information about on COVID-19, perceived reduction of noise pollution, and COVID-19 infection.

Sleep features module included the Pittsburgh Sleep Quality Index (PSQI, used to evaluate sleep quality, the score ranges from 0 to 21 , a higher score is associated with a worst condition), variation of sleep time duration, perceived variation of SQ, and variation of sleep latency.

Psychological module was composed by Beck Depression Inventory (BDI, measures the severity of depression, score ranges from 0 to 63 , a higher score is associated with a worst condition), State-Trait Anxiety Inventory (STAI, evaluates anxiety through two different score, one for the trait anxiety, one for the state anxiety, each one ranges from 20 to 80 , and a higher score is associated with a higher anxiety level), variation in perceived anxiety/depression, Perceived Stress scale (PSS, assesses perceived stress, it ranges from 0 to 40, and a higher score is associated with higher stress perception), variation in perceived stress, concern for the future in lockdown, variation of concern for the future, times a day to go outside, and concern for COVID-19.

Migraine module evaluated migraine familiarity, anti-migraine drug overuse story, migraine with aura, age of onset, age of migraine chronification, variation of migraine frequency (increased, reduced, or a stable number of migraine days per month compared to pre-lockdown period), variation of migraine attack duration (increase, reduction, or no change compared to pre-lockdown period), increased migraine pain intensity during lockdown, variation of migraine symptomatic drugs use per week (increase, reduction, unchanged in comparison with previous period), variation of migraine drug efficacy (increase, reduction, unchanged compared to previous period), the six-item headache impact test (HIT-6, provides a global measure of adverse headache impact, the score ranges from 36 to 78 , a higher score is associated with a worst condition).

Every patient had an own migraine diary and was asked to respond to frequency, duration, intensity, and symptomatic drug use questions according to it.

Using our headache center archive, we also verified history of anti-migraine drug overuse and evaluated the discontinuation of the therapy performed within the headache center (botulinum toxin or monoclonal antibodies) due to lockdown. It should be noted that only headache centers were authorized to provide monoclonal antibodies acting on the CGRP pathway until the end of July 2020 and our center could not do it during the lockdown period.

\section{Study outcomes}

Every collected variable was referred to the following outcomes:

- Migraine impact on daily life (HIT-6)

- Variation of migraine frequency (number of migraine days per month)

- Variation of migraine attack duration

- Increased migraine pain intensity 
- Variation of migraine symptomatic drugs use per week

- Variation of migraine drug efficacy

\section{Ethics}

The research was conducted ethically in accordance with the World Medical Association Declaration of Helsinki. The study protocol has been approved by the local research institute's committee on human research. All the patients have given their written informed consent.

\section{Statistical analysis}

All statistical analyses were performed using $\mathrm{R}$ software. Continuous variables were expressed as mean \pm standard deviation; categorical variables were expressed as absolute frequencies and percentages. Continuous variables were analyzed by Shapiro-Wilk test to evaluate normal distribution. Mann-Whitney $U$ or Student's $t$ test for independent samples was used for comparison between categorical variables with two levels and continuous variables as appropriate. ANOVA test or Kruskal-Wallis test was used for comparison between categorical variables with $>2$ levels and continuous variables on the basis of normal distribution. The chi-square test was used for comparison between categorical variables. The method of partitioning the degrees of freedom was applied to refuse $\mathrm{H}_{0}$ hypothesis as appropriate. Spearman's rank or Pearson's correlation coefficient was used for comparison between continuous variables as appropriate.

The multivariate analysis was performed using the multiple logistic regression model. Regarding outcomes with three levels (variation of migraine frequency, variation of migraine attack duration, variation of migraine symptomatic drug use, and variation of migraine drug efficacy), we built two different models. In the first model, "improved" and "no change" categories were unified; in the second model, "worsened" and "no change" categories were unified in order to perform multivariate analysis. A value of $P<0.05$ was considered significant.

\section{Results}

Among 150 chronic migraineurs followed in our headache center, 92 patients accepted to participate in the study.

A migraine familial history was present in $80,4 \%$ of respondents. Age of migraine onset was $\leq 18$ years in $66,3 \%$. Migraine became chronic at an age $\leq 18$ years in $27,2 \%$, between 18 and 30 years in $41,3 \%$, and at an age $\geq 31$ years in $31,5 \%$. Aura was present in $8,7 \%$ of patients. An antimigraine drug overuse story was present in $80,4 \%$. Patients had a HIT-6 score of $64,63 \pm 8,81$.

Migraine attack frequency was stable in $40,2 \%$, increased $33,7 \%$, and reduced in $26,1 \%$; migraine attack duration was stable in $55,4 \%$, increased in $23,9 \%$, and reduced in $20,7 \%$ (Fig. 1). Migraine pain was stable or reduced in $65,2 \%$ and increased in $34,8 \%$; number of migraine symptomatic drugs per week was stable in $50 \%$, reduced in $29,3 \%$, and increased in $20,7 \%$; migraine drug efficacy was stable in $73,9 \%$, reduced in $17,4 \%$, and increased in $8,7 \%$. Migraine data are reported in Table 1.

Demographic, life style, sleep, and psychological data are reported in Tables 2 and 3.

\section{Influences of demographics, life style, sleep, psychological, and migraine features on migraine outcome}

\section{HIT-6}

A higher HIT-6 score was associated with low educational qualifications, unemployment, more hours of television viewing, a reduction in meal regularity, worsening in SQ, a
Fig. 1 Chronic migraine changes during lockdown

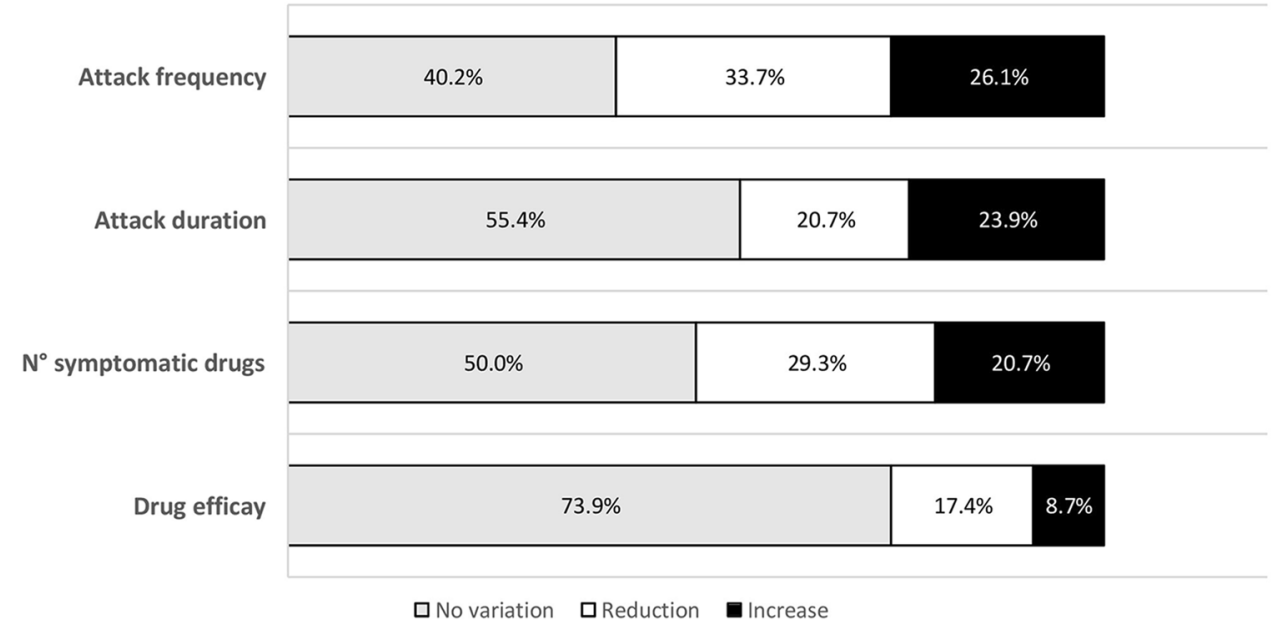


Table 1 Migraine related data

\begin{tabular}{ll}
\hline & $N(\%)$ \\
\hline Familiarity & $74(80,4 \%)$ \\
Age of onset $\leq 18$ years & $61(66,3 \%)$ \\
Age of chronification & \\
$\leq 18$ years & $25(27,2 \%)$ \\
18-30 years & $38(41,3 \%)$ \\
$\geq 31$ years & $29(31,5 \%)$ \\
Aura & $8(8,7 \%)$ \\
Migraine drug overuse & $74(80,4 \%)$ \\
Discontinuation of therapy performed within center & $13(14,1 \%)$ \\
& $M e a n \pm \mathrm{DS}$ \\
HIT-6 & $64,63 \pm 8,81$ \\
& $N(\%)$ \\
Attack frequency variation & \\
No variation & $37(40,2 \%)$ \\
Decrease & $24(26,1 \%)$ \\
Increase & $31(33,7 \%)$ \\
Attack duration change & \\
No change & $51(55,4 \%)$ \\
Decrease & $19(20,7 \%)$ \\
Increase & $22(23,9 \%)$ \\
Increased pain & $32(34,8 \%)$ \\
Symptomatic drugs per week variation & \\
No change & $46(50,0 \%)$ \\
Increase & $27(29,3 \%)$ \\
Migraine drug efficacy variation & $19(20,7 \%)$ \\
No change & \\
Decrease & $68(73,9 \%)$ \\
\hline & $16(17,4 \%)$ \\
\hline Increase & $8,7 \%)$ \\
\hline
\end{tabular}

HIT-6, six-item headache impact test

higher BDI score, worsening in perceived depression/anxiety, a higher State-Trait Anxiety Inventory-State (STAI-S) score, a higher State-Trait Anxiety Inventory-Trait (STAIT) score and a higher PSS score (Table S1).

On multivariate analysis, only low educational qualification and a higher STAI-T remained significant.

\section{Variation of the number of migraine days per month}

An increased migraine attack frequency was associated with smoke, reduced sleep time duration, reduced quality of sleep, increased sleep latency, higher PSQI, higher BDI, worsening in perceived depression/anxiety, increased perceived stress, STAI-S, and STAI-T (Table 4). On multivariate analysis, only smoke and a high STAI-S were associated with increased frequency.

\section{Variation of migraine attack duration}

An increased migraine attack duration was associated with a reduction in meal regularity, reduced sleep time duration, reduced quality of sleep, increased sleep latency, worsening in perceived depression/anxiety, a higher PSS score, and increased perceived stress. A reduced migraine attack duration was associated with RW. Both increase and reduction of migraine attack duration were associated with longer internet use time. See Table 4. Multivariate analysis confirmed that the decrease was related with RW and the increment was associated with reduced sleep duration and a higher PSS score.

\section{Variation of migraine pain intensity}

An increased migraine pain intensity was associated with lower ratio of house size/number of people, longer smartphone use time, longer internet use time, worsening in meal quality, a reduction in meal regularity, concern for the future, reduced sleep time duration, reduced quality of sleep, higher PSQI score, no migraine familiarity, worsening in perceived depression/anxiety, increased perceived stress, higher BDI score, higher STAI-S score, higher STAI-T score, and higher PSS score (Table 4). Concern for the future, reduced sleep time duration, no migraine familiarity, increased perceived stress, and higher STAI-T score remained significant on multivariate analysis.

\section{Variation of migraine symptomatic drug use per week}

An increased migraine symptomatic drugs use per week was associated with discontinuation of the therapy performed within headache center, reduced quality of sleep, worsening in perceived depression/anxiety, increased perceived stress, and higher STAI-S score (Table 5). Only discontinuation of the therapy performed within headache center and STAI-S was confirmed on multivariate analysis.

\section{Variation of migraine drug efficacy}

A reduction of migraine drug efficacy was associated with smoke, increased sleep latency, worsening in perceived depression/anxiety, increased perceived stress, higher STAI$\mathrm{S}$ score, and concern for COVID-19. An increased migraine drug efficacy was associated with RW and an improved quality of sleep. Both increase and reduction in migraine drug efficacy were associated with an increase in cigarette consumption. See Table 5. Multivariate analysis showed that the efficacy reduction was associated with smoke, STAI-S, and concern for COVID-19 and that the improvement was related with remote working and improved quality of sleep. 
Table 2 Demographic and lifestyle data

\begin{tabular}{|c|c|c|}
\hline & $N$ & $(\%)$ \\
\hline Gender: Female & 79 & $(85,9 \%)$ \\
\hline Age: $\leq 40$ years old & 39 & $(42,24 \%)$ \\
\hline \multicolumn{3}{|l|}{ Educational qualification } \\
\hline Primary/secondary school graduation & 25 & $(27,2 \%)$ \\
\hline High school graduation & 46 & $(50 \%)$ \\
\hline Degree/post graduate education & 21 & $(22,8 \%)$ \\
\hline \multicolumn{3}{|l|}{ Unemployment } \\
\hline Yes & 32 & $(34,8 \%)$ \\
\hline No & 60 & $(65,2 \%)$ \\
\hline Stop to work/study & 19 & $(20,7 \%)$ \\
\hline Remote working & 20 & $(21,7 \%)$ \\
\hline Job loss & 8 & $(8,7 \%)$ \\
\hline Home size $\leq 100 \mathrm{sqm}$ & 42 & $(45,7 \%)$ \\
\hline Living with other people & 83 & $(90,2 \%)$ \\
\hline Ratio of house size/number of people $\leq 40$ sqm per person & 58 & $(63,0 \%)$ \\
\hline Computer Hours $\geq 5$ & 28 & $(30,4 \%)$ \\
\hline \multicolumn{3}{|l|}{ Variation computer hours } \\
\hline No variation & 43 & $(46,7 \%)$ \\
\hline Fewer & 13 & $(14,1 \%)$ \\
\hline More & 36 & $(39,1 \%)$ \\
\hline Smartphone hours $\geq 5$ & 23 & $(25,0 \%)$ \\
\hline \multicolumn{3}{|l|}{ Variation smartphone hours } \\
\hline No variation & 29 & $(31,5 \%)$ \\
\hline Fewer & 6 & $(6,5 \%)$ \\
\hline More & 57 & $(62,0 \%)$ \\
\hline Internet hours $\geq 5$ & 22 & $(23,9 \%)$ \\
\hline \multicolumn{3}{|l|}{ Variation internet hours } \\
\hline No variation/fewer & 44 & $(47,8 \%)$ \\
\hline More & 48 & $(52,2 \%)$ \\
\hline Television hours $\geq 5$ & 13 & $(14,1 \%)$ \\
\hline \multicolumn{3}{|l|}{ Variation television hours } \\
\hline No variation /fewer & 47 & $(51,1 \%)$ \\
\hline More & 45 & $(48,9 \%)$ \\
\hline \multicolumn{3}{|l|}{ Meal quality } \\
\hline Same & 46 & $(50,0 \%)$ \\
\hline Worsening & 26 & $(28,3 \%)$ \\
\hline Improvement & 20 & $(21,7 \%)$ \\
\hline \multicolumn{3}{|l|}{ Meal regularity } \\
\hline Same & 54 & $(58,7 \%)$ \\
\hline Worsening & 21 & $(22,8 \%)$ \\
\hline Improvement & 17 & $(18,5 \%)$ \\
\hline Smoker & 22 & $(23,9 \%)$ \\
\hline \multicolumn{3}{|l|}{ Smoke variation } \\
\hline No variation/reduction & 77 & $(83,7 \%)$ \\
\hline Increase & 15 & $(16,3 \%)$ \\
\hline \multicolumn{3}{|l|}{ Coffee cups per day } \\
\hline No & 23 & $(25,0 \%)$ \\
\hline$\leq 2$ & 39 & $(42,4 \%)$ \\
\hline$>3$ & 30 & $(32,6 \%)$ \\
\hline \multicolumn{3}{|l|}{ Coffee consume variation } \\
\hline No variation & 67 & $(72,8 \%)$ \\
\hline
\end{tabular}


Table 2 (continued)

\begin{tabular}{lll}
\hline & $N$ & $(\%)$ \\
\hline Less & 11 & $(12,0 \%)$ \\
More & 14 & $(15,0 \%)$ \\
Sons/daughters & & \\
No sons/daughters & 45 & $(48,9 \%)$ \\
Sons/daughters $<18$ years & 20 & $(21,7 \%)$ \\
Rent/mortgage & 30 & $(32,6 \%)$ \\
Home-inhabitant relationship & & $(44,6 \%)$ \\
Good & 41 & $(42,4 \%)$ \\
Very good & 39 & $(13,0 \%)$ \\
No good & 12 & $(27,2 \%)$ \\
Living with parents & 25 & $(38,0 \%)$ \\
Time to focus on the news about COVID-19>2 times a day & 35 & $(63,0 \%)$ \\
COVID-19 province prevalence $>0,0632$ cases per population $(\%)$ & 58 & $(00,0 \%)$ \\
COVID-19 infection & 0 & $(28,3 \%)$ \\
Going out during the lockdown & & $(56,5 \%)$ \\
Never & & $(15,2 \%)$ \\
$1-2$ times a day & 26 & $(89,1 \%)$ \\
3 or more times a day & 52 & 14 \\
Reduction in noise pollution & 82 & \\
\hline
\end{tabular}

\section{Discussion}

During lockdown, our patients responded in a different manner: approximately half had a clinical stability, a quarter had a migraine improvement, and the other quarter a worsening compared to the pre-lockdown month. In detail, the migraine frequency was stable in $40,2 \%$, increased in $26,1 \%$, and reduced in $33,7 \%$; the attack duration was unchanged in $55,4 \%$, increased in $23,9 \%$, and reduced in $20,7 \%$; migraine pain was stable or reduced in $65,2 \%$ and intensified in $34,8 \%$. Number of migraine symptomatic drugs per week was the same in $50 \%$, reduced in $29,3 \%$, and increased in $20,7 \%$; migraine drug efficacy was stable in $73,9 \%$, reduced in $17,4 \%$, and increased in $8,7 \%$. Patients had a HIT-6 score of $64,63 \pm 8,81$.

In the present study, migraine severity and changes in lockdown were associated with several elements: some classical migraine-related factors and others that were never reported in literature.

Low educational qualification (LEQ), a well-known risk factor for CM [9], was associated with higher HIT-6 score suggesting which part of our migraineurs are more vulnerable. Around life style, our smoker patients showed an increased migraine attack frequency and a reduction of migraine drug efficacy. Smoke is, indeed, related in different studies with migraine and constitutes an important headache trigger $[5,10]$.

Anxiety, perceived stress, and sleep have a significant influence in our patients. High level of anxiety was linked with all examined outcomes. Anxiety disorders are, indeed, very common in migraine, two to five times more prevalent than in the general population, and they are much more common in patients with $\mathrm{CM}$ than episodic migraine [11] and were also associated with more severe migraine [12]. The perceived stress in our patients was linked with attack duration and pain intensity. Stress during lockdown, in line with the literature, certainly had a determinant role in our patients' worsening. Stress is a prevalent migraine trigger and it is also considered to exacerbate and maintain migraine [11, 13]. Major life events are related with headache chronification [14] and perceived stress was related with CM in Moon et al. study [15]. Anxiety and perceived stress in migraineurs are important signs of fragility to take into consideration to avoid migraine worsening. We specifically investigated concerns about future and COVID19: they were associated with pain intensity and reduced drug efficacy, respectively. This was in line with anxiety and stressful status. Regarding sleep, the present study showed that a reduced sleep time duration was related with an increment in migraine attack duration and pain. A sleep quality improvement was also associated with an increased drug efficacy. Sleep is, indeed, another important factor which influences CM: high attack frequency had been related with poor SQ and poor sleepers; CM had been associated with non-restorative sleep, poor sleep habits, short sleep time, and longer sleep latency [16]. Our results reaffirm as sleep has a key role in this disease and is influenced by life changes. The sleep problems, together with anxiety and stress, should be always investigated in migraineurs and treated in collaboration with other professional figures 
Table 3 Psychological and sleep related data

\begin{tabular}{|c|c|c|}
\hline & $N$ & $(\%)$ \\
\hline \multicolumn{3}{|l|}{ State Anxiety (STAI-S) } \\
\hline Average anxiety (41-60) & 50 & $(54,3 \%)$ \\
\hline Above average anxiety $(61-100)$ & 23 & $(25,0 \%)$ \\
\hline Below average anxiety $(0-40)$ & 19 & $(20,7 \%)$ \\
\hline \multicolumn{3}{|l|}{ Trait Anxiety (STAI-T) } \\
\hline Average anxiety (41-60) & 46 & $(50 \%)$ \\
\hline Above average anxiety $(61-100)$ & 17 & $(18,5 \%)$ \\
\hline Below average anxiety $(0-40)$ & 29 & $(31,5 \%)$ \\
\hline \multicolumn{3}{|l|}{ Depression (BDI) } \\
\hline Average (0-13) & 59 & $(64,1 \%)$ \\
\hline Moderate (14-28) & 24 & $(26,1 \%)$ \\
\hline Severe (29-63) & 9 & $(9,8 \%)$ \\
\hline \multicolumn{3}{|l|}{ Anxiety/depression variation } \\
\hline No variation & 51 & $(55,4 \%)$ \\
\hline Reduction & 9 & $(9,8 \%)$ \\
\hline Increase & 32 & $(34,8 \%)$ \\
\hline \multicolumn{3}{|l|}{ Future concern } \\
\hline No or low & 14 & $(15,2 \%)$ \\
\hline Medium & 45 & $(48,9 \%)$ \\
\hline High & 33 & $(35,9 \%)$ \\
\hline Future concern increase & 54 & $(58,7 \%)$ \\
\hline COVID-19 concern & 76 & $(82,6 \%)$ \\
\hline \multicolumn{3}{|l|}{ Perceived stress (PSS) } \\
\hline Low & 14 & $(15,2 \%)$ \\
\hline Moderate & 57 & $(62,0 \%)$ \\
\hline High & 21 & $(22,8 \%)$ \\
\hline \multicolumn{3}{|l|}{ Stress variation } \\
\hline No variation & 34 & $(37,0 \%)$ \\
\hline Reduction & 13 & $(14,1 \%)$ \\
\hline Increase & 45 & $(48,9 \%)$ \\
\hline \multicolumn{3}{|l|}{ Sleep time variation } \\
\hline No variation & 32 & $(34,8 \%)$ \\
\hline Reduction & 27 & $(29,3 \%)$ \\
\hline Increase & 33 & $(35,9 \%)$ \\
\hline \multicolumn{3}{|l|}{ Sleep quality variation } \\
\hline No variation & 44 & $(47,8 \%)$ \\
\hline Worsening & 35 & $(38,0 \%)$ \\
\hline Improvement & 13 & $(14,1 \%)$ \\
\hline \multicolumn{3}{|l|}{ Sleep latency } \\
\hline No variation & 43 & $(46,7 \%)$ \\
\hline Reduction & 5 & $(5,4 \%)$ \\
\hline \multirow[t]{2}{*}{ Increase } & 44 & $(47,8 \%)$ \\
\hline & Mean \pm SD & \\
\hline PSQI & $11,96 \pm 5,85$ & \\
\hline
\end{tabular}

STAI-S, State-Trait Anxiety Inventory-State; STAI-T, State-Trait Anxiety Inventory-Trait; $B D I$, Beck Depression Inventory; PSS, Perceived Stress scale; PSQI, Pittsburgh Sleep Quality Index

such as sleep specialists, psychologists, and psychiatrists in order to improve patients' quality of life.
A controversial point is the association between no migraine family history and increased pain intensity. Familial predisposition plays an important role in migraine: it was linked with an increased migraine risk and a higher attack frequency in other studies [17]. A possible explanation of our findings could be that no-familial forms are more influenced by external elements and life changes than familial forms. Regarding treatment with botulinum toxin and monoclonal antibodies, it was stopped during the lockdown and our study showed that the discontinuation led to an increase in migraine symptomatic drug consumption. The therapy discontinuation led also a worsening in other outcomes but the small size of the population examined probably did not permit to obtain a statistical significance.

An interesting finding is that RW was associated with reduced migraine attack duration and increased drug efficacy. RW has progressively spread in recent years, but its use is enormously increased during the lockdown due to COVID-19, allowing to maintain different service ensuring the worker safety. No other studies reported a link between RW and migraine, probably because they evaluated mainly migraine frequency. We hypothesize that this improvement could be attributed to the distance from workplace and its stressor, and the possibility to manage time in a different manner. Previous studies indicated time flexibility as a main strong point of RW, and it allows the people to shape the work on the basis of their needs [18]. This is particularly relevant for migraineur who could avoid exposure to factors that could favor, worse, and prolong the migraine attack. RW was associated with better performance, more satisfaction, reduced stress, less absenteeism, and more motivation in several studies [18]. It should be taken in consideration in order to ameliorate the condition of subjects afflicted by chronic migraine that represent a frail class of workers. RW and time flexibility could also increase level of employment in these patients that often give up working because of their condition. Specific studies are needed to evaluate the effect of RW in migraineurs workers and in particular outside of pandemic and lockdown context to verify our findings in normal everyday life.

Several studies evaluated migraine in the COVID19 period. However, the present investigation is the only one focused on CM patients. The other studies associated migraine changes with sleep disturbance, depression, anxiety, emotional reaction, pandemic risk perception, computer use, eating habits, and physical activity during lockdown [19-27]. It is interesting to observe the different trends in these studies: the majority of Al Hashel et al. patients had a worsening [19]; most patients were stable in Smith et al. study [25]; Delussi et al., Parodi et al., and Verhagen et al. migraineurs had an improvement [20,21, 26]; and the majority of Dallavalle et al. patients improved or were stable on the basis of pre-lockdown condition [22]. Gentile 


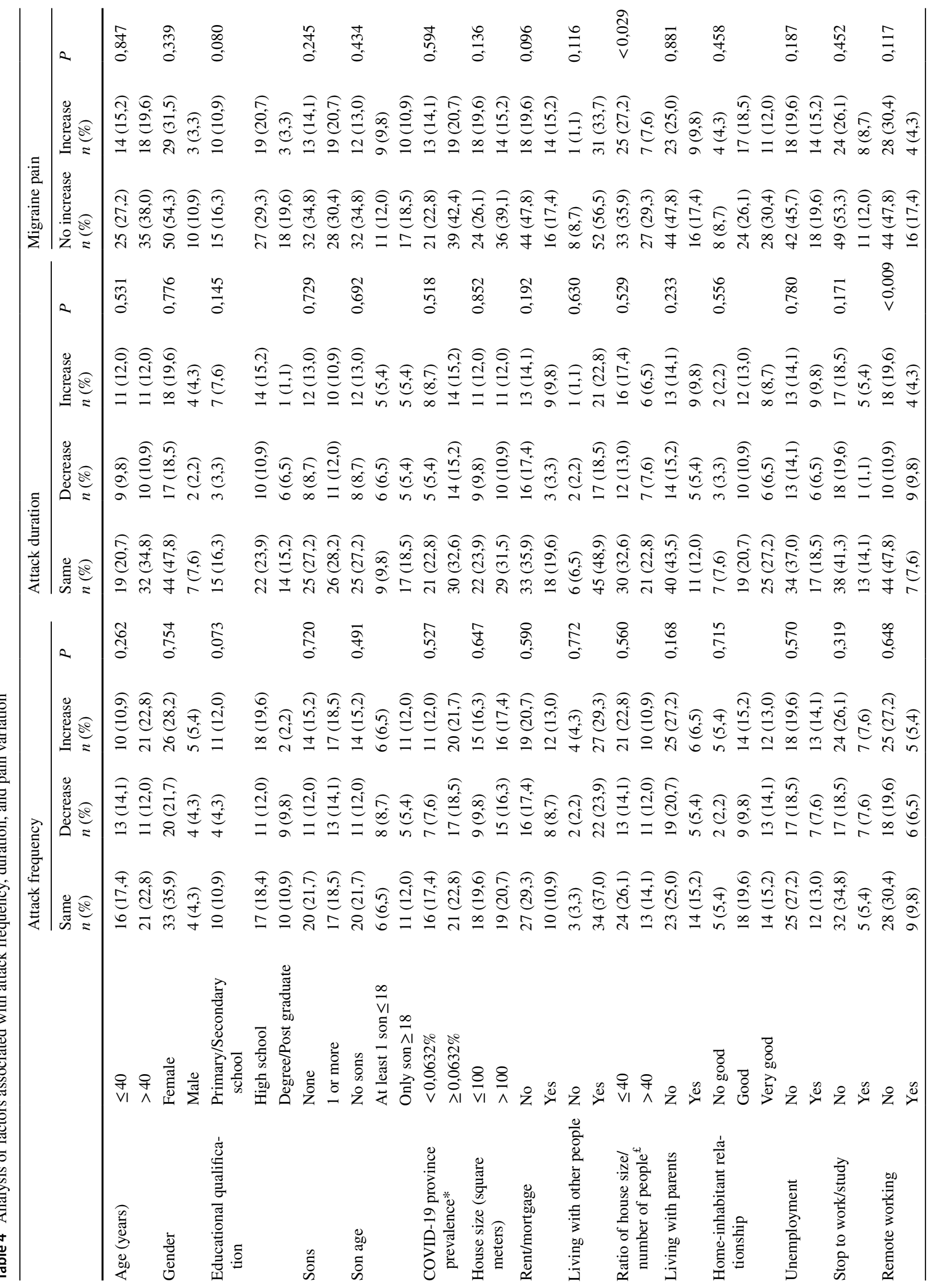




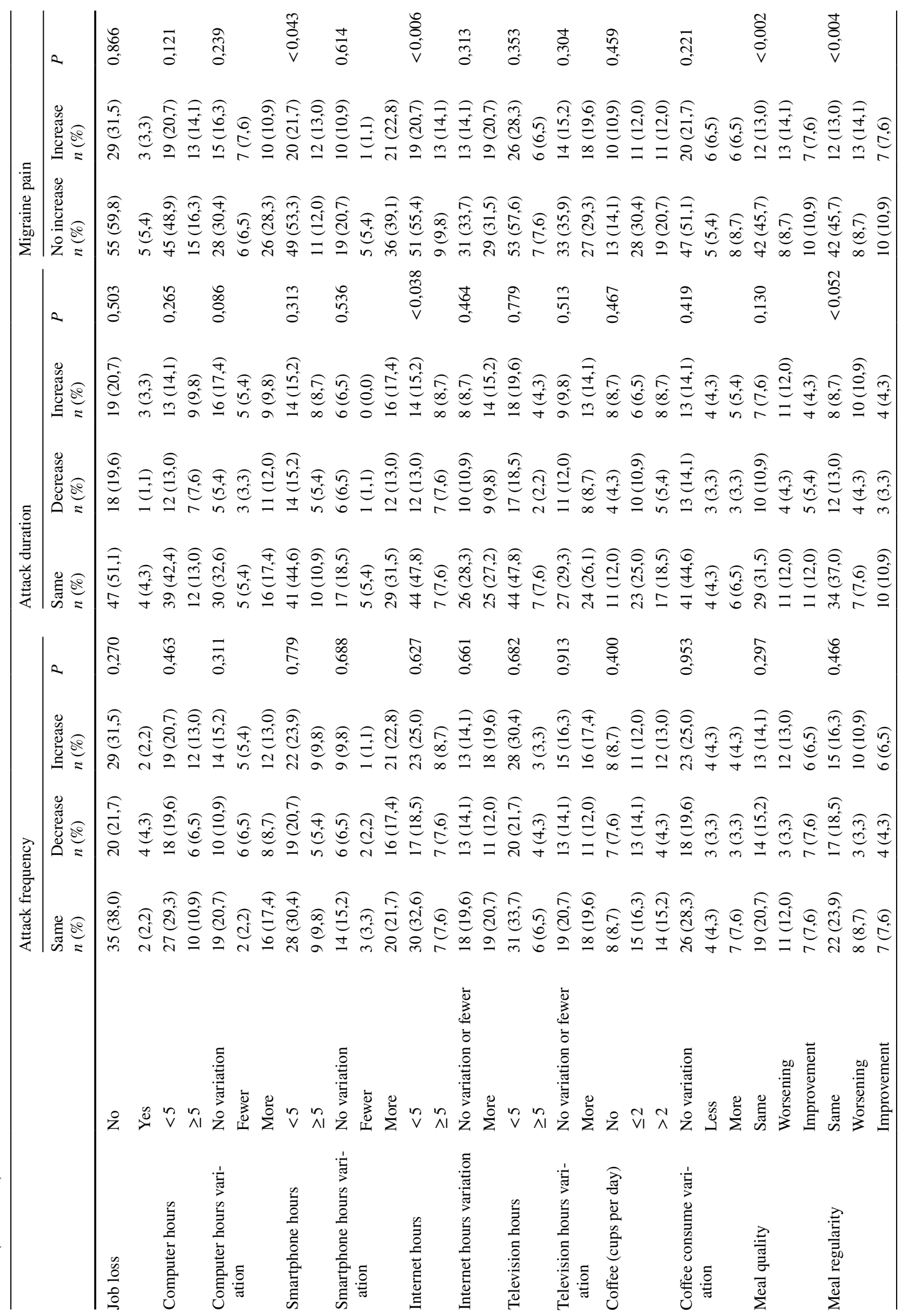




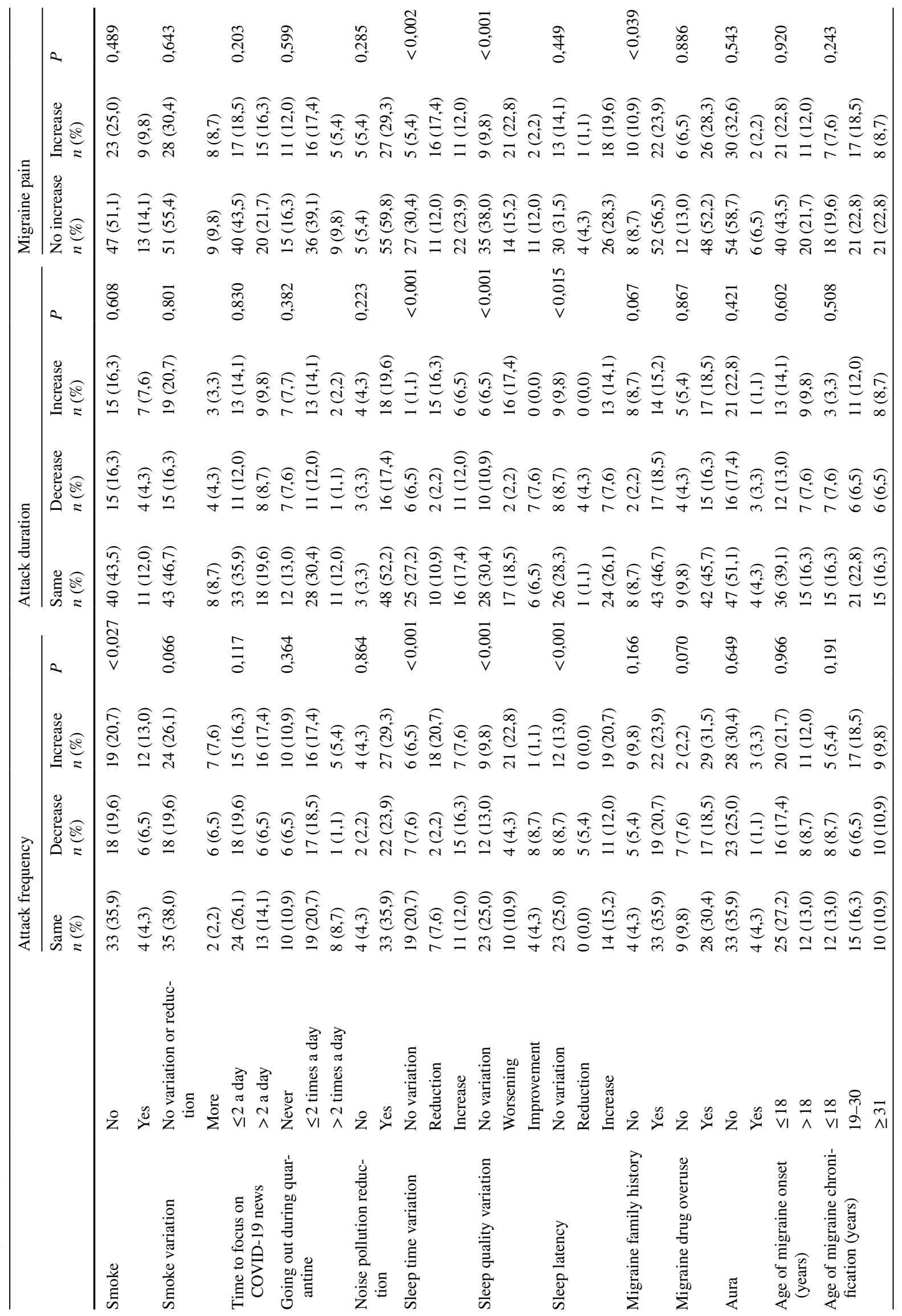




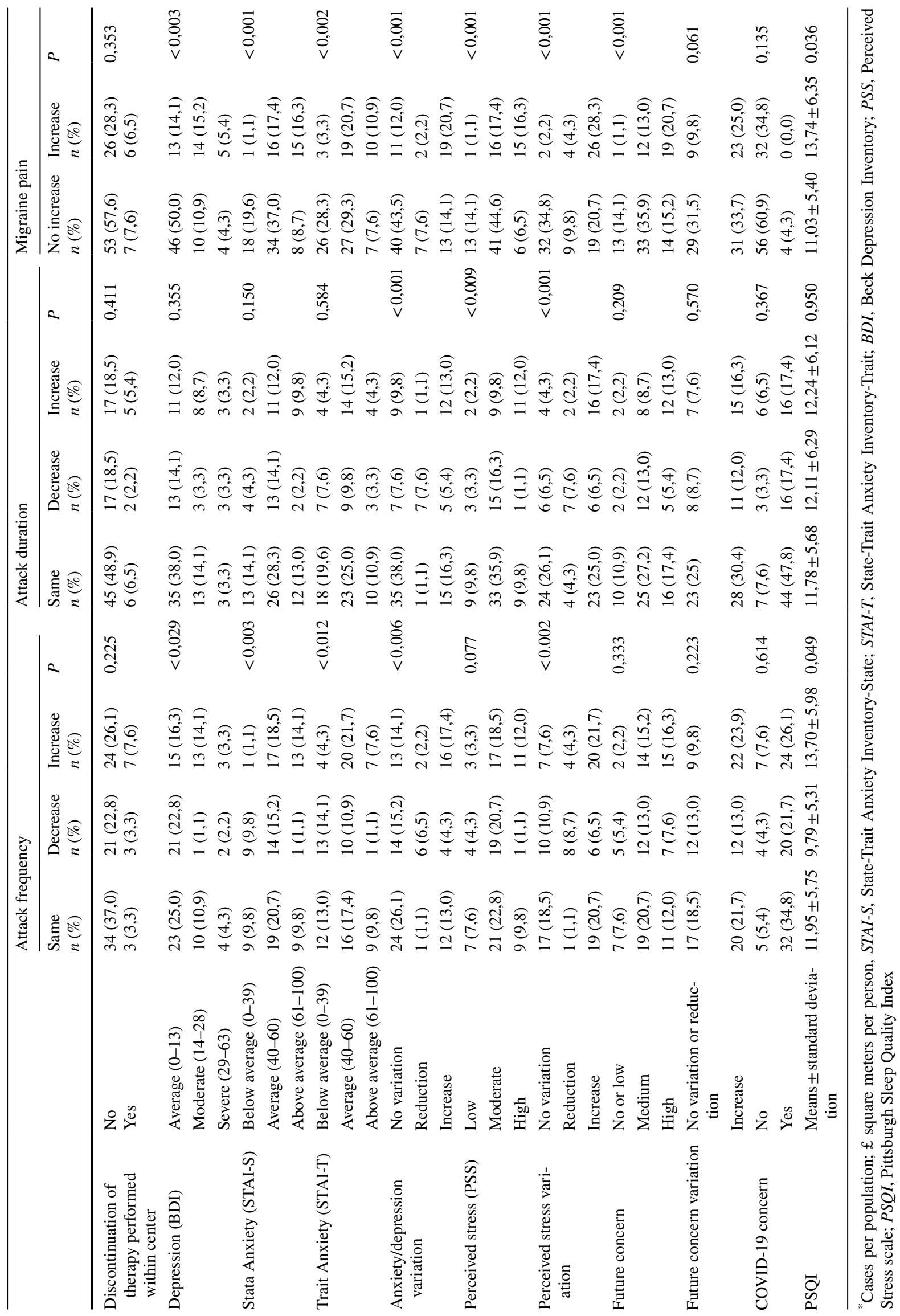


Table 5 Symptomatic drugs per week and efficacy variation between previous month and lockdown

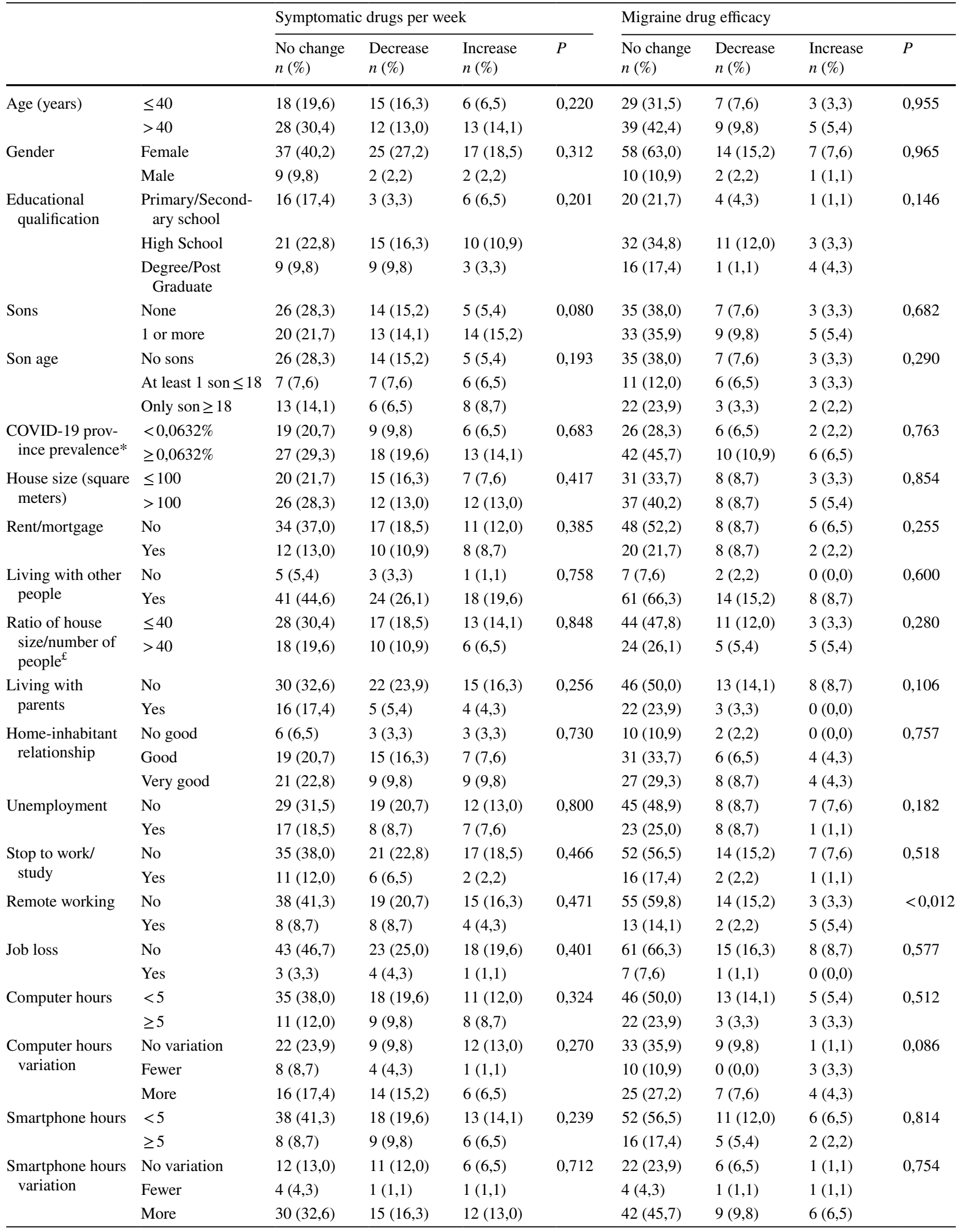


Table 5 (continued)

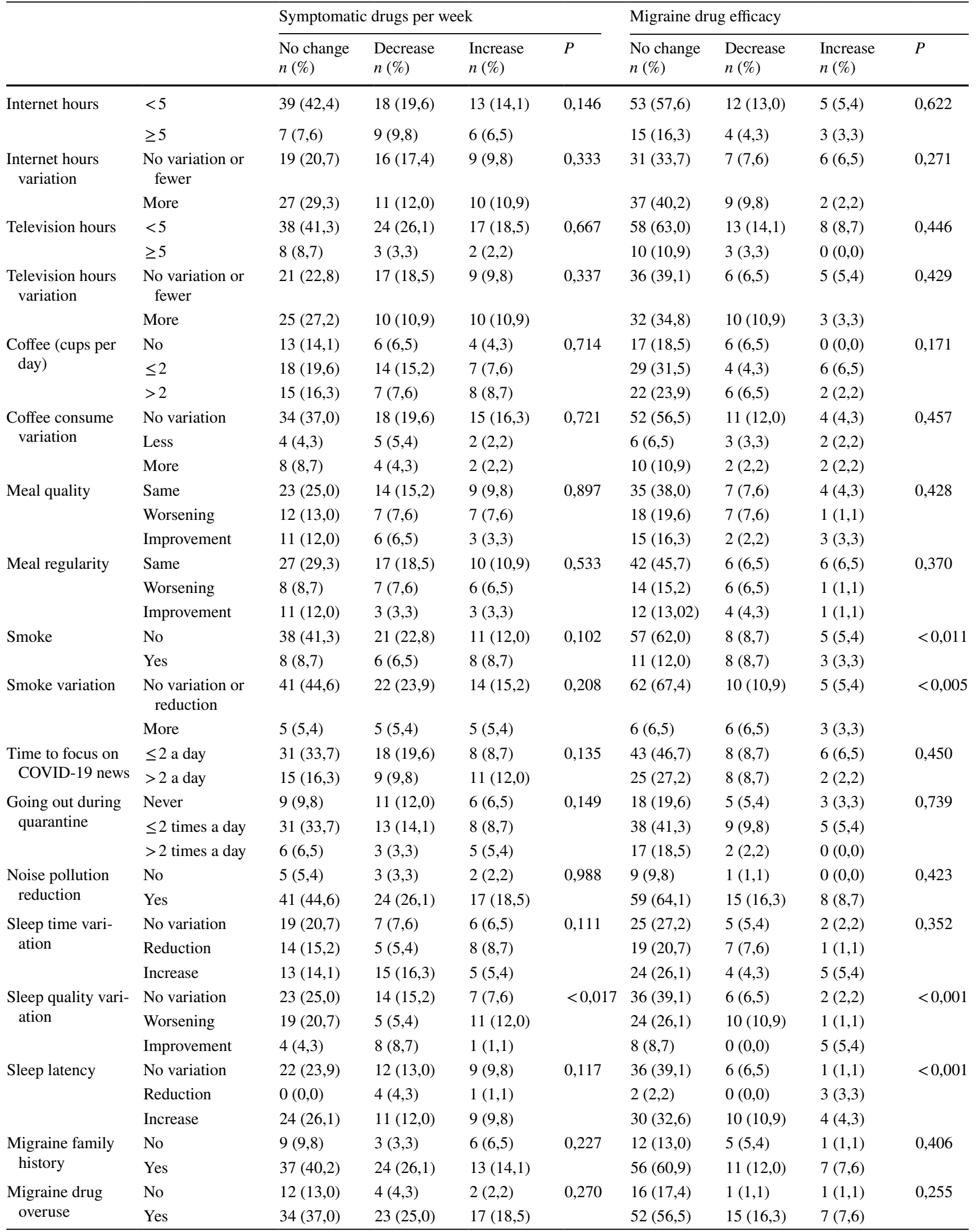


Table 5 (continued)

\begin{tabular}{|c|c|c|c|c|c|c|c|c|c|}
\hline & \multicolumn{4}{|c|}{ Symptomatic drugs per week } & \multicolumn{4}{|c|}{ Migraine drug efficacy } \\
\hline & & $\begin{array}{l}\text { No change } \\
n(\%)\end{array}$ & $\begin{array}{l}\text { Decrease } \\
n(\%)\end{array}$ & $\begin{array}{l}\text { Increase } \\
n(\%)\end{array}$ & $P$ & $\begin{array}{l}\text { No change } \\
n(\%)\end{array}$ & $\begin{array}{l}\text { Decrease } \\
n(\%)\end{array}$ & $\begin{array}{l}\text { Increase } \\
n(\%)\end{array}$ & $P$ \\
\hline \multirow[t]{2}{*}{ Aura } & No & $42(45,7)$ & $25(27,2)$ & $17(18,5)$ & 0,934 & $61(66,3)$ & $15(16,3)$ & $8(8,7)$ & 0,577 \\
\hline & Yes & $4(4,3)$ & $2(2,2)$ & $2(2,2)$ & & $7(7,6)$ & $1(1,1)$ & $0(0,0)$ & \\
\hline \multirow{2}{*}{$\begin{array}{l}\text { Age of migraine } \\
\text { onset (years) }\end{array}$} & $\leq 18$ & $29(31,5)$ & $19(20,7)$ & $13(14,1)$ & 0,796 & $46(50,0)$ & $10(10,9)$ & $5(5,4)$ & 0,900 \\
\hline & $>18$ & $17(18,5)$ & $8(8,7)$ & $6(6,5)$ & & $22(23,9)$ & $6(6,5)$ & $3(3,3)$ & \\
\hline \multirow{3}{*}{$\begin{array}{l}\text { Age of migraine } \\
\text { chronification } \\
\text { (years) }\end{array}$} & $\leq 18$ & $10(10,9)$ & $12(13,0)$ & $3(3,3)$ & 0,108 & $20(21,7)$ & $3(3,3)$ & $2(2,2)$ & 0,676 \\
\hline & $19-30$ & $20(21,7)$ & $7(7,6)$ & $11(12,0)$ & & $28(30,4)$ & $8(8,7)$ & $2(2,2)$ & \\
\hline & $\geq 31$ & $16(17,4)$ & $8(8,7)$ & $5(5,4)$ & & $20(21,7)$ & $5(5,4)$ & $4(4,3)$ & \\
\hline \multirow{2}{*}{$\begin{array}{l}\text { Discontinuation } \\
\text { of therapy per- } \\
\text { formed within } \\
\text { center }\end{array}$} & No & $42(45,7)$ & $25(27,2)$ & $12(13,0)$ & 0,006 & $60(65,2)$ & $12(13,0)$ & $7(7,6)$ & 0,389 \\
\hline & Yes & $4(4,3)$ & $2(2,2)$ & $7(7,6)$ & & $8(8,7)$ & $4(4,3)$ & $1(1,1)$ & \\
\hline \multirow[t]{3}{*}{ Depression (BDI) } & Average (0-13) & $31(33,7)$ & $19(20,7)$ & $9(9,8)$ & 0,243 & $46(50,0)$ & $6(6,5)$ & $7(7,6)$ & 0,078 \\
\hline & Moderate (14-28) & $12(13,0)$ & $4(4,3)$ & $8(8,7)$ & & $17(18,5)$ & $7(7,6)$ & $0(0,0)$ & \\
\hline & Severe (29-63) & $3(3,3)$ & $4(4,3)$ & $2(2,2)$ & & $5(5,4)$ & $3(3,3)$ & $1(1,1)$ & \\
\hline \multirow[t]{3}{*}{$\begin{array}{c}\text { Stata Anxiety } \\
\text { (STAI-S) }\end{array}$} & $\begin{array}{l}\text { Below average } \\
(0-39)\end{array}$ & $13(14,1)$ & $6(6,5)$ & $0(0,0)$ & $<0,012$ & $16(17,4)$ & $0(0,0)$ & $3(3,3)$ & $<0,044$ \\
\hline & Average (40-60) & $25(27,2)$ & $26(28,3)$ & $9(9,8)$ & & $38(41,3)$ & $8(8,7)$ & $4(4,3)$ & \\
\hline & $\begin{array}{l}\text { Above average } \\
(61-100)\end{array}$ & $8(8,7)$ & $5(5,4)$ & $10(10,9)$ & & $14(15,2)$ & $8(8,7)$ & $1(1,1)$ & \\
\hline \multirow[t]{3}{*}{$\begin{array}{l}\text { Trait Anxiety } \\
\text { (STAI-T) }\end{array}$} & $\begin{array}{l}\text { Below average } \\
(0-39)\end{array}$ & $18(19,6)$ & $10(10,9)$ & $1(1,1)$ & 0,100 & $22(23,9)$ & $2(2,2)$ & $5(5,4)$ & 0,126 \\
\hline & Average (40-60) & $21(22,8)$ & $12(13,0)$ & $13(14,1)$ & & $35(38,0)$ & $9(9,8)$ & $2(2,2)$ & \\
\hline & $\begin{array}{l}\text { Above average } \\
(61-100)\end{array}$ & $7(7,6)$ & $5(5,4)$ & $5(5,4)$ & & $11(12,0)$ & $5(5,4)$ & $1(1,1)$ & \\
\hline \multirow{3}{*}{$\begin{array}{l}\text { Anxiety/depres- } \\
\text { sion variation }\end{array}$} & No variation & $32(34,8)$ & $12(13,0)$ & $7(7,6)$ & $<0.009$ & $40(43,5)$ & $7(7,6)$ & $4(4,4)$ & $<0,018$ \\
\hline & Reduction & $2(2,2)$ & $6(6,5)$ & $1(1,1)$ & & $6(6,5)$ & $0(0,0)$ & $3(3,3)$ & \\
\hline & Increase & $12(13,0)$ & $9(9,8)$ & $11(12,0)$ & & $22(23,9)$ & $9(9,8)$ & $1(1,1)$ & \\
\hline \multirow{3}{*}{$\begin{array}{l}\text { Perceived stress } \\
\text { (PSS) }\end{array}$} & Low & $8(8,7)$ & $4(4,3)$ & $2(2,2)$ & 0,490 & $9(9,8)$ & $2(2,2)$ & $3(3,3)$ & 0,062 \\
\hline & Moderate & $28(30,4)$ & $19(20,7)$ & $10(10,9)$ & & $45(48,9)$ & $7(7,6)$ & $5(5,4)$ & \\
\hline & High & $10(10,9)$ & $4(4,3)$ & $7(7,6)$ & & $14(15,2)$ & $7(7,6)$ & $0(0,0)$ & \\
\hline \multirow{3}{*}{$\begin{array}{l}\text { Perceived stress } \\
\text { variation }\end{array}$} & No variation & $22(23,9)$ & $9(9,8)$ & $3(3,3)$ & $<0.030$ & $28(30,4)$ & $4(4,3)$ & $2(2,2)$ & $<0,001$ \\
\hline & Reduction & $4(4,3)$ & $7(7,6)$ & $2(2,2)$ & & $8(8,7)$ & $0(0,0)$ & $5(5,4)$ & \\
\hline & Increase & $20(21,7)$ & $11(12,0)$ & $14(15,2)$ & & $32(34,8)$ & $12(13,0)$ & $1(1,1)$ & \\
\hline \multirow[t]{3}{*}{ Future concern } & No or low & $10(10,9)$ & $3(3,3)$ & $1(1,1)$ & 0,124 & $12(13,0)$ & $1(1,1)$ & $1(1,1)$ & 0,406 \\
\hline & Medium & $22(23,9)$ & $16(17,4)$ & $7(7,6)$ & & $35(38,0)$ & $6(6,5)$ & $4(4,3)$ & \\
\hline & High & $14(15,2)$ & $8(8,7)$ & $11(12,0)$ & & $21(22,8)$ & $9(9,8)$ & $3(3,3)$ & \\
\hline \multirow[t]{2}{*}{$\begin{array}{l}\text { Future concern } \\
\text { variation }\end{array}$} & $\begin{array}{l}\text { No variation or } \\
\text { reduction }\end{array}$ & $21(22,8)$ & $11(12,0)$ & $6(6,5)$ & 0,576 & $29(31,5)$ & $6(6,5)$ & $3(3,3)$ & 0,908 \\
\hline & Increase & $25(27,2)$ & $16(17,4)$ & $13(14,1)$ & & $39(42,4)$ & $10(10,9)$ & $5(5,4)$ & \\
\hline \multirow{2}{*}{$\begin{array}{l}\text { COVID-19 con- } \\
\text { cern }\end{array}$} & No & $8(8,7)$ & $3(3,3)$ & $5(5,4)$ & 0,408 & $10(10,9)$ & $6(6,5)$ & $0(0,0)$ & $<0,038$ \\
\hline & Yes & $38(41,3)$ & $24(26,1)$ & $14(15,2)$ & & $58(63,0)$ & $10(10,9)$ & $8(8,7)$ & \\
\hline PSQI & $\begin{array}{l}\text { Means } \pm \text { standard } \\
\text { deviation }\end{array}$ & $11,50 \pm 5,78$ & $11,41 \pm 5,91$ & $13,94 \pm 5,84$ & 0,276 & $11,61 \pm 5,67$ & $14,19 \pm 5,94$ & $10,38 \pm 6,74$ & 0,209 \\
\hline
\end{tabular}

*Cases per population; $£$ square meters per person; STAI-S, State-Trait Anxiety Inventory-State; STAI-T, State-Trait Anxiety Inventory-Trait; BDI, Beck Depression Inventory; SS, Perceived Stress scale; PSQI, Pittsburgh Sleep Quality Index

et al. showed a migraine worsening during the second lockdown [23]. Di Stefano et al. reported that one-third of the patients were stable, one-third had a worsening, and the remaining an improvement [27]. Focusing on the Italian first lockdown and on adult patients with CM, our study did not show the improvement that was present in Altamura 
et al. and Delussi et al. chronic migraineurs. Altamura et al. patients' improvement was most probably due to monoclonal antibody administration. The differences with Delussi et al. could be explained through the different interview time: our survey was started on April $24^{\text {th }}$ and was closed on May $3^{\text {rd }}$, and theirs between March $27^{\text {th }}$ and April $18^{\text {th }}$. Delussi et al. attributed the improvement to patients' resilience [21] that could have been eroded by time, justifying the different results.

There are several limitations in our study. The first limitation is the small number of patients. Second, non-response from the web-based survey may result in selection bias.

Third, we do not have standardized data in the pre-lockdown period, and we are based on patients' report, migraine diary, and perception. Fourth, many outcomes and variables taken in consideration have subjective characteristics and are prone to recall bias that are common in these types of studies. Fifth, the study in a single institution may have affected the selection of patients.

\section{Conclusion}

During lockdown, our patients responded in a different manner: approximately half had a clinical stability, a quarter had a migraine improvement, and the remaining a worsening. Our study represented a unique prospective to observe and evaluate CM in different conditions from daily routine. Differently than other studies, we focused on CM patients, the migraineurs who are frailest and the most difficult to treat. We found some elements which represented vulnerability points that must be evaluated in migraine. Anxiety, stress, and sleep problems represent an enormous burden for CM that negatively influence their life and would be always investigated and treated in collaboration with different professional figures.

The most relevant study finding is the improvement due to the remote working; it could represent an easy way to ameliorate the condition of chronic migraineurs, increasing both their well-being and work performance.

Supplementary Information The online version of this article contains supplementary material available (https://doi.org/10.1007/ s10072-021-05521-7).

Author contribution CCT, ESV, and AC contributed to the study design; CCT, AC, and CV performed the data collection; TG performed the statistical analysis; AT, MA, GV, and RS supervised the research; CCT, AC, CV, RS, and MA wrote the article.

Availability of data and material Derived data supporting the findings of this study are available from the corresponding author on request.

\section{Declarations}

Conflict of interest The authors declare no competing interests.

Ethical approval The research was conducted ethically in accordance with the World Medical Association Declaration of Helsinki. The study protocol has been approved by the research institute's committee on human research.

Consent to participate and for publication All the patients have given their written informed consent.

\section{References}

1. Stovner LJ, Nichols E, Steiner TJ et al (2018) Global, regional, and national burden of migraine and tension-type headache, 1990-2016: a systematic analysis for the Global Burden of Disease Study 2016. Lancet Neurol 17:954-976. https://doi.org/10. 1016/S1474-4422(18)30322-3

2. Leonardi M, Raggi A (2013) Burden of migraine: international perspectives. Neurol Sci 34:117-118. https://doi.org/10.1007/ s10072-013-1387-8

3. Nowaczewska M, Wiciński M, Kaźmierczak W (2020) The ambiguous role of caffeine in migraine headache: from trigger to treatment. Nutrients. https://doi.org/10.3390/nu12082259

4. Bigal ME, Lipton RB (2006) Modifiable risk factors for migraine progression. Headache 46:1334-1343. https://doi.org/10.1111/j. 1526-4610.2006.00577.x

5. Kelman L (2007) The triggers or precipitants of the acute migraine attack. Cephalalgia 27:394-402. https://doi.org/10.1111/j.14682982.2007.01303.x

6. Montagni I, Guichard E, Carpenet C et al (2016) Screen time exposure and reporting of headaches in young adults: a crosssectional study. Cephalalgia 36:1020-1027. https://doi.org/10. 1177/0333102415620286

7. Seidel S, Hartl T, Weber M et al (2009) Quality of sleep, fatigue and daytime sleepiness in migraine-a controlled study. Cephalalgia 29:662-669. https://doi.org/10.1111/j.1468-2982.2008. 01784.x

8. Karthik N, Kulkarni GB, Taly AB et al (2012) Sleep disturbances in "migraine without aura" - a questionnaire based study. J Neurol Sci 321:73-76. https://doi.org/10.1016/j.jns.2012.07.057

9. May A, Schulte LH (2016) Chronic migraine: risk factors, mechanisms and treatment. Nat Rev Neurol 12:455-464. https://doi.org/ 10.1038/nrneurol.2016.93

10. Schramm SH, Obermann M, Katsarava Z et al (2013) Epidemiological profiles of patients with chronic migraine and chronic tension-type headache. J Headache Pain 14:1-8. https://doi.org/ 10.1186/1129-2377-14-40

11. Minen MT, De Dhaem OB, Van Diest AK et al (2016) Migraine and its psychiatric comorbidities. J Neurol Neurosurg Psychiatry 87:741-749. https://doi.org/10.1136/jnnp-2015-312233

12. Tomé-Pires $\mathrm{C}$, Solé E, Racine $\mathrm{M}$ et al (2016) The relative importance of anxiety and depression in pain impact in individuals with migraine headaches. Scand J Pain 13:109-113. https://doi.org/10. 1016/j.sjpain.2016.08.002

13. Wacogne C, Lacoste JP, Guillibert E et al (2003) Stress, anxiety, depression and migraine. Cephalalgia 23:451-455. https://doi.org/ 10.1046/j.1468-2982.2003.00550.x

14. Scher AI, Midgette LA, Lipton RB (2008) Risk factors for headache chronification. Headache 48:16-25. https://doi.org/10.1111/j. 1526-4610.2007.00970.x 
15. Moon HJ, Seo JG, Park SP (2017) Perceived stress in patients with migraine: a case-control study. J Headache Pain. https://doi.org/ 10.1186/s10194-017-0780-8

16. Yang CP, Wang SJ (2017) Sleep in patients with chronic migraine. Curr Pain Headache Rep 21:1-7. https://doi.org/10.1007/ s11916-017-0641-9

17. De Boer I, Van Den Maagdenberg AMJM, Terwindt GM (2019) Advance in genetics of migraine. Curr Opin Neurol 32:413-421. https://doi.org/10.1097/WCO.0000000000000687

18. De Macêdo TAM, Cabral ELDS, Silva Castro WR et al (2020) Ergonomics and telework: a systematic review. Work 66:777-788. https://doi.org/10.3233/WOR-203224

19. Al-Hashel JY, Ismail II (2020) Impact of coronavirus disease 2019 (COVID-19) pandemic on patients with migraine: a web-based survey study. J Headache Pain 21:115. https://doi.org/10.1186/ s10194-020-01183-6

20. Parodi IC, Poeta MG, Assini A et al (2020) Impact of quarantine due to COVID infection on migraine: a survey in Genova, Italy. Neurol Sci 41:2025-2027. https://doi.org/10.1007/ s10072-020-04543-x

21. Delussi M, Gentile E, Coppola G et al (2020) Investigating the effects of COVID-19 quarantine in migraine: an observational cross-sectional study from the Italian National Headache Registry (RICe). Front Neurol 11:1-8. https://doi.org/10.3389/fneur.2020. 597881

22. Dallavalle G, Pezzotti E, Provenzi L et al (2020) Migraine symptoms improvement during the COVID-19 lockdown in a cohort of children and adolescents. Front Neurol 11:1-6. https://doi.org/ 10.3389/fneur.2020.579047

23. Gentile E, Delussi M, Abagnale C et al (2021) Brain sciences migraine during COVID-19: data from second wave pandemic in an Italian cohort. 1-9

24. Altamura C, Cevoli S, Aurilia C et al (2020) Locking down the CGRP pathway during the COVID-19 pandemic lockdown: the PandeMig study. Neurol Sci 41:3385-3389. https://doi.org/10. 1007/s10072-020-04767-x

25. Smith M, Nakamoto M, Crocker J et al (2020) Early impact of the COVID-19 pandemic on outpatient migraine care in Hawaii: results of a quality improvement survey. Headache J Head Face Pain. https://doi.org/10.1111/head.14030

26. Verhagen IE, van Casteren DS, Terwindt L, de Gisela MS, V, (2020) Effect of lockdown during Covid-19 on migraine: a cohort study. Cephalalgia 40:10. https://doi.org/10.1177/0333102420 981739

27. Di Stefano V, Ornello R, Gagliardo A et al (2021) Social distancing in chronic migraine during the covid-19 outbreak: results from a multicenter observational study. Nutrients 13:1-13. https://doi. org/10.3390/nu13041361

Publisher's note Springer Nature remains neutral with regard to jurisdictional claims in published maps and institutional affiliations. 